\title{
Study of methods for dimension reduction of complex dynamic linear systems models
}

\author{
Yuriy M. Manatskov ${ }^{1 *}$, Torsten Bertram ${ }^{2}$, Danil V. Shaykhutdinov ${ }^{1}$ \\ and Nikolay I. Gorbatenko ${ }^{1}$ \\ ${ }^{1}$ Platov South-Russian State Polytechnic University (NPI), Information Measuring Systems and \\ Technologies Department, 346428 Novocherkassk, Russia \\ ${ }^{2} \mathrm{TU}$ Dortmund, Department of Electrical Engineering and Information Technology, 44227 Dortmund, \\ Germany
}

\begin{abstract}
Complex dynamic linear systems of equations are solved by numerical iterative methods, which need much computation and are timeconsuming ones, and the optimization stage requires repeated solution of these equation systems that increases the time on development. To shorten the computation time, various methods can be applied, among them preliminary (estimated) calculation or oversimple models calculation, however, while testing and optimizing the full model is used. Reduced order models are very popular in solving this problem. The main idea of a reduced order model is to find a simplified model that may reflect the required properties of the original model as accurately as possible. There are many methods for the model order reduction, which have their advantages and disadvantages. In this article, a method based on Krylov subspaces and SVD methods is considered. A numerical experiments is given.
\end{abstract}

\section{Introduction}

Currently, simulation is a must-do step while developing, designing and optimizing new objects or their units. Objects simulation allows minimizing the stages of setting up and testing, as well as time and money costs. Simulation makes it possible to test and optimize individual physical units of the system, replacing the entire system with a model. Today, automatic simulation packages, such as Ansys, Comsol Mutiphysics MATLAB / SIMULINK, Proteus, Microcap etc. for various fields of science and technology have been developed. Each package uses individual computational algorithms, which are becoming more complex, allowing solving increasingly complicated problems. For example, thanks to the development of computing power, the finite element method (FEM) has become very popular nowadays that is a powerful tool for performing complex engineering calculations. Meanwhile, as the algorithms and objects to be simulated are becoming more complex, there appears a difficulty in testing and optimizing the model. This is due to huge number of equations inevitably accompanying the calculations.

\footnotetext{
* Corresponding author: manackov25@gmail.com
} 
As a rule, such systems of equations are solved by numerical iterative methods, which need much computation and are time-consuming ones, and the optimization stage requires repeated solution of these equation systems that increases the time on development. To shorten the computation time, various methods can be applied, among them preliminary (estimated) calculation or oversimple models calculation, however, while testing and optimizing the full model is used.

Now, reduced order models are very popular in solving this problem [1-3]. The main idea of a reduced order model is to find a simplified model that may reflect the required properties of the original model as accurately as possible. There are many methods for the model order reduction, which have their advantages and disadvantages. In this article, a method based on Krylov subspaces and SVD methods will be considered.

\section{The problem setting}

The task is to identify and reduce the number of variables in the space of system states of a given dimension.

Before applying the methods for order reduction, it is necessary to determine which type a system belongs to: SISO (simple input - simple output) or MIMO (multi input - multi output), linear or non-linear. At present, the algorithms for reduction of the linear systems order that satisfy a user's needs are developed, but the work for non-linear systems is still in process. For simplicity, we will consider a linear system SISO (one input - one output); the same methods may be converted to satisfy the MIMO systems.

Let us consider the system as:

$$
\mathbf{G}:\left\{\begin{array}{l}
\dot{\mathbf{x}}(t)=\mathbf{A}_{n \times n^{\mathbf{x}}(t)+\mathbf{B}_{n \times p}} \mathbf{u}(t) \\
\mathbf{y}(t)=\mathbf{C}_{q \times n} \mathbf{x}(t)+\mathbf{D}_{q \times p} \mathbf{u}(t)
\end{array}\right.
$$

where $\mathbf{A}_{n \times n}$ - system matrix, $\mathbf{B}_{n \times p}$ - control matrix, $\mathbf{C}_{q \times n}-$ output matrix, $\mathbf{D}_{q \times p}-$ feedback matrix (usually equal to 0 that says about absence of direct feedback), $\mathbf{x}(t)-$ state vector, $\mathbf{y}(t)$ - output vector, $\mathbf{u}(t)-$ control vector.

After applying the order reduction methods, the system is transformed to the form:

$$
\mathbf{G}_{r}:\left\{\begin{array}{l}
\dot{\mathbf{x}}_{r}(t)=\mathbf{A}_{r} \mathbf{x}_{r}(t)+\mathbf{B}_{r} \mathbf{u}(t) \\
\mathbf{y}(t)=\mathbf{C}_{r} \mathbf{x}_{r}(t)+\mathbf{D u}(t)
\end{array}\right.
$$

where $\mathbf{A}_{r}, \mathbf{B}_{r}, \mathbf{C}_{r}, \mathbf{x}_{r}$ - reduced matrices of the system, control, output and state vector, respectively.

This operation can be represented graphically, as shown in Fig. 1.

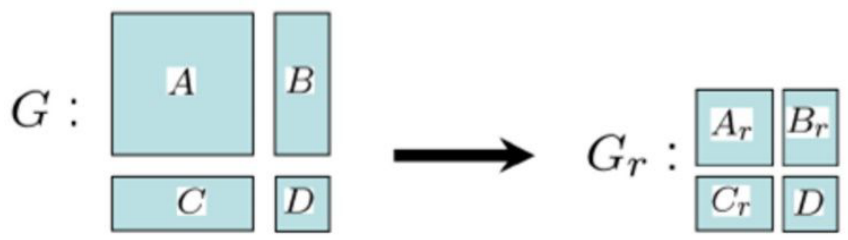

Fig 1. Transformation of the complete model into a reduced order model. 
To obtain the reduced order models of linear systems, various methods are used: methods for coordination of the transfer function moments (based on Krylov subspaces) and SDVdecomposition methods. Figure 2 shows a subdivision of order reduction methods

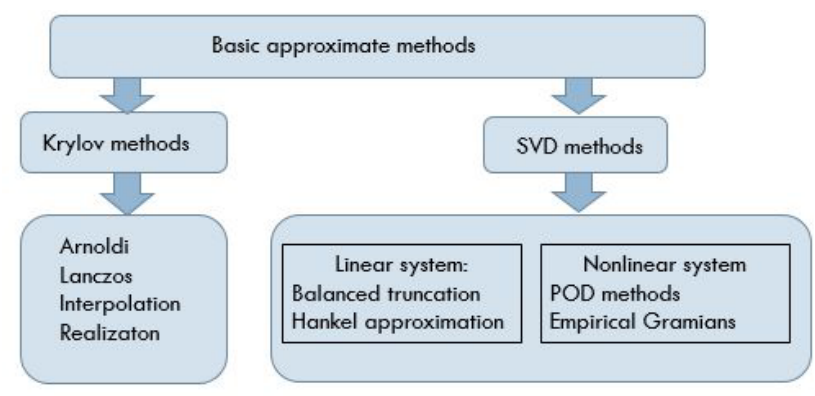

Fig. 2. Methods of order reduction of linear systems.

\section{Krylov subspace methods}

The order reduction methods based on Krylov subspaces refer to finite element methods. The basic idea of model order reduction is that the vector $\mathrm{x}$ behavior can be described by some subspace $\mathbf{x}_{r}$ of small dimension. Then, $\mathbf{x}=\mathbf{V} \mathbf{x}_{r}$, where $\mathbf{V}$ is the transformation matrix (projection basis). Consider the initial linear system (1), which can be described by the transfer function in the frequency domain:

$$
s \mathbf{x}^{\prime}=\mathbf{A x}(s)+\mathbf{B u}, \quad \mathbf{y}(s)=\mathbf{C}^{T} \mathbf{x}(s), \quad \mathbf{H}(s)=\frac{\mathbf{Y}(s)}{\mathbf{U}(s)} .
$$

For the linear model represented by the transfer function, one of the most direct methods of obtaining a reduced order model is possible - the matching of the reduced and complete transfer functions [4]. Here, there are several methods, for example, Padé approximation, asymptotic vibration estimation method (AWE) and biorthogonalization method (PWL).

If we neglect the mathematical proof, then after applying the methods of order reduction, taking into account the Krylov subspaces and using the matrices of transformation $-\mathbf{W}$ and conversion $-\mathbf{V}[5]$, the original system is transformed to the form:

$$
\mathbf{G}_{r}:\left\{\begin{array}{l}
\dot{\mathbf{x}}_{r}(t)=\mathbf{A}_{r} \mathbf{x}_{r}(t)+\mathbf{B}_{r} \mathbf{u}(t) \\
\mathbf{y}(t)=\mathbf{C}_{r} \mathbf{x}_{r}(t)+\mathbf{D u}(t)
\end{array}\right.
$$

where $\mathbf{A}_{r}=\mathbf{V}^{T} \mathbf{A W}, \mathbf{B}_{r}=\mathbf{V}^{T} \mathbf{B}, \mathbf{C}_{r}=\mathbf{C W}, \mathbf{D}=\mathbf{D}$.

To calculate the matrices $\mathbf{W}$ and $\mathbf{V}$, the following algorithms are used:

1) the orthogonal Arnoldi algorithm [6] when $\mathbf{W}=\mathbf{V}$ and the matrix $\mathbf{W}$ is orthogonal, i.e. $\mathbf{W}^{T} \mathbf{V}=\mathbf{I}_{r}$, then:

$$
\mathbf{K}_{r}(\mathbf{A}, \mathbf{B})=\operatorname{span}\left\{\mathbf{B}, \mathbf{A B}, \mathbf{A}^{2} \mathbf{B}, \ldots, \mathbf{A}^{r-1} \mathbf{B}\right\}, \mathbf{W}=\operatorname{span}\left[\mathbf{B}, \mathbf{A B}, \mathbf{A}^{2} \mathbf{B}, \ldots, \mathbf{A}^{r-1} \mathbf{B}\right],
$$

where span - Krylov subspace; 
2) Lanczos algorithm, when $\mathbf{W}$ and $\mathbf{V}$ matrices are biorthogonal, i.e. $\mathbf{V}^{T} \mathbf{W}=\mathbf{I}_{r}$, then:

$$
\begin{gathered}
\mathbf{K}_{r}(\mathbf{A}, \mathbf{B})=\operatorname{span}\left\{\mathbf{B}, \mathbf{A B}, \mathbf{A}^{2} \mathbf{B}, \ldots, \mathbf{A}^{r-1} \mathbf{B}\right\}, \mathbf{W}=\operatorname{span}\left[\mathbf{B}, \mathbf{A B}, \mathbf{A}^{2} \mathbf{B}, \ldots, \mathbf{A}^{r-1} \mathbf{B}\right], \\
\mathbf{K}_{r}\left(\mathbf{A}^{T}, \mathbf{C}^{T}\right)=\operatorname{span}\left\{\mathbf{C}, \mathbf{A}^{T} \mathbf{C}^{T},\left(\mathbf{A}^{T}\right)^{2} \mathbf{C}^{T}, \ldots,\left(\mathbf{A}^{T}\right)^{r-1} \mathbf{C}^{T}\right\}, \\
\mathbf{V}=\operatorname{span}\left[\mathbf{C}^{T}, \mathbf{A}^{T} \mathbf{C}^{T},\left(\mathbf{A}^{T}\right)^{2} \mathbf{C}^{T}, \ldots,\left(\mathbf{A}^{T}\right)^{r-1} \mathbf{C}^{T}\right] .
\end{gathered}
$$

\section{SVD - methods}

SVD methods are based on the singular value decomposition (SVD), which allows calculating the singular matrix numbers, as well as right and left singular vectors. Each of the singular numbers estimates the influence of an element on the general behavior of the system [4].

Before applying SVD methods, first the original system is decomposed into stable and unstable subsystems. Further, order reduction methods are applied to its stable subsystem; eventually, both subsystems are combined into one general reduced order model [6], as shown in Figure 3.

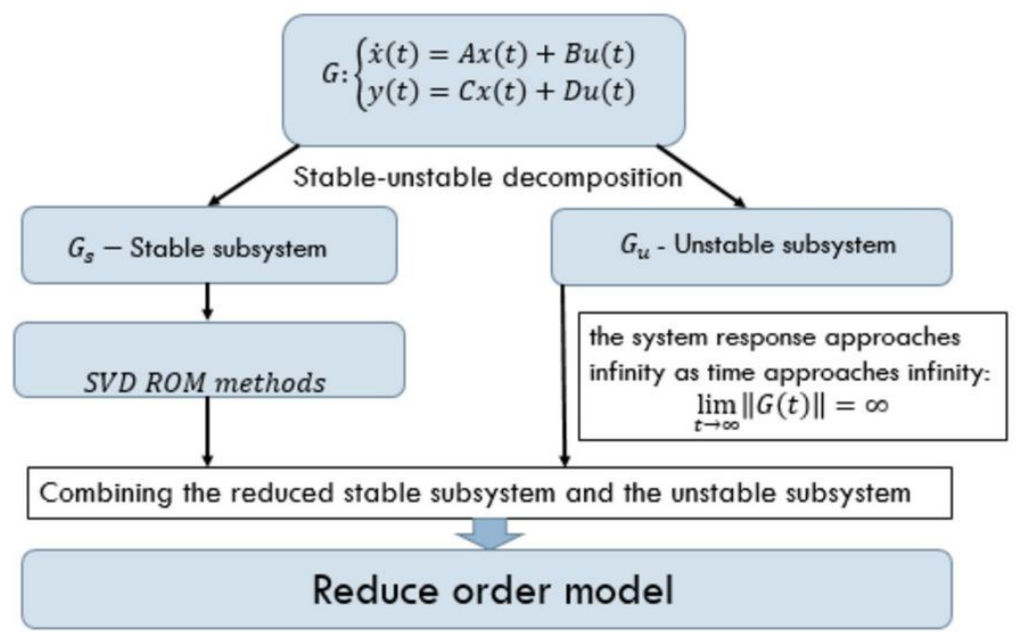

Fig. 3. Algorithm for order reducing of the unstable systems.

\section{Truncation balancing method}

The main idea of the method is that some states of the system are difficult to observe, while others are difficult to control. Balancing truncation allows saving only well-controlled and observed states by removing uncontrolled and non-observed states.

Consider the linear system (1); let the matrix $\mathbf{A}$ be asymptotically stable, then by solving the Lyapunov equations system, the observability gramian $\mathbf{W}_{\mathbf{o}}$ and the controllability gramian $\mathbf{W}_{\mathbf{c}}$ can be calculated:

$$
\mathbf{A}^{T} \mathbf{W}_{o}+\mathbf{W}_{o} \mathbf{A}+\mathbf{C}^{T} \mathbf{C}=0, \mathbf{A} \mathbf{W}_{c}+\mathbf{W}_{c} \mathbf{A}^{T}+\mathbf{B B} \mathbf{B}^{T}=0 .
$$


Operations of this type are realized in MATLAB (Wc=gram(sys,'c'), W0=gram(sys,'o'))

After balancing the system using the function in MATLAB ([sysb, $\sigma, \mathrm{Ti}, \mathrm{T}]=$ balreal(sys)), the gramians of controllability and observability have the form:

$$
\mathbf{W}_{c}=\mathbf{W}_{o}=\Sigma, \Sigma=\left(\begin{array}{ccc}
\sigma_{1} & \ldots & 0 \\
\vdots & \ddots & \vdots \\
0 & \ldots & \sigma_{n}
\end{array}\right)
$$

The singular values in matrix $\boldsymbol{\Sigma}$ are arranged in decreasing order. If we remove small singular numbers whose order is much less than the value of the first singular number, we will get a new reduced order system, as shown below [7]:

$$
\begin{aligned}
& \hat{\mathbf{W}}_{c}=\hat{\mathbf{W}}_{o}=\left(\begin{array}{cc}
\Sigma_{1} & 0 \\
0 & \Sigma_{2}
\end{array}\right) \text { if } \Sigma_{1}>\Sigma_{2}, \\
& \mathbf{G}:\left\{\begin{array}{l}
\dot{\mathbf{x}}(t)=\left(\begin{array}{ll}
\mathbf{A}_{11} & \mathbf{A}_{12} \\
\mathbf{A}_{21} & \mathbf{A}_{22}
\end{array}\right) \mathbf{x}(t)+\left(\begin{array}{c}
\mathbf{B}_{1} \\
\mathbf{B}_{2}
\end{array}\right) \mathbf{u}(t) ; \\
\mathbf{y}(t)=\left(\begin{array}{l}
\mathbf{C}_{1} \\
\mathbf{E}_{2}
\end{array}\right) \mathbf{x}(t),
\end{array}\right. \\
& \mathbf{G}_{r}:\left\{\begin{array}{l}
\dot{\mathbf{x}}(t)=\mathbf{A}_{11} \mathbf{x}(t)+\mathbf{B}_{1} \mathbf{u}(t) ; \\
\mathbf{y}(t)=\mathbf{C}_{1} \mathbf{x}(t) .
\end{array}\right.
\end{aligned}
$$

An important feature of the method is the possibility to control the error:

$$
\delta \leq 2 \underset{i=q+1}{\sum_{i} \sigma^{i}}
$$

\section{Hankel approximation method}

The Hankel approximation method is similar to the balanced truncation method [5] but it allows obtaining more reliable reduced order models. Consider the linear system (1), its matrices $\mathbf{A}, \mathbf{B}, \mathbf{C}, \mathbf{D}$ and the order of the required reduced order model $\mathrm{k}$. The first stage falls with the one within the balanced truncation method where it is necessary to calculate the observability gramian $\mathbf{W}_{\mathbf{o}}$ and the controllability gramian $\mathbf{W}_{\mathbf{c}}$. Then, a descriptor is generated:

$$
\mathbf{E}=\mathbf{W}_{c} \mathbf{W}_{o}-\rho^{2} \mathbf{I}
$$

where $\sigma_{k}>\rho>>\sigma_{k+1}$

We apply SDV-decomposition to the descriptor and divide the result by the $\mathrm{k}^{\text {th }}$ order to obtain:

$$
\mathbf{E}=\left[\begin{array}{ll}
\mathbf{U}_{E 1} & \mathbf{U}_{E 2}
\end{array}\right] \cdot\left[\begin{array}{cc}
\Sigma_{E} & 0 \\
0 & 0
\end{array}\right] \cdot\left[\begin{array}{c}
\mathbf{V}_{E 1}^{T} \\
\mathbf{V}_{E 2}^{T}
\end{array}\right],\left[\begin{array}{cc}
\mathbf{E} s-\overline{\mathbf{A}} & \overline{\mathbf{B}} \\
\overline{\mathbf{C}} & \overline{\mathbf{D}}
\end{array}\right]=\left[\begin{array}{cc}
\rho^{2} \mathbf{A}^{T}-\mathbf{W}_{o} \mathbf{A} \mathbf{W}_{c} & \mathbf{W}_{o} \mathbf{B} \\
\mathbf{W}_{c} \mathbf{C} & \mathbf{D}
\end{array}\right]
$$

Inserting the descriptor in the state space matrices, we get the following representation of the model: 


$$
\begin{gathered}
{\left[\begin{array}{ll}
\mathbf{A}_{11} & \mathbf{A}_{12} \\
\mathbf{A}_{21} & \mathbf{A}_{22}
\end{array}\right]=\left[\begin{array}{c}
\mathbf{U}_{E 1}^{T} \\
\mathbf{U}_{E 2}^{T}
\end{array}\right]\left(\rho^{2} \mathbf{A}^{T}+\mathbf{W}_{o} \mathbf{A} \mathbf{W}_{c}\right)\left[\begin{array}{ll}
\mathbf{V}_{E 1} & \mathbf{V}_{E 2}
\end{array}\right],} \\
{\left[\begin{array}{l}
\mathbf{B}_{1} \\
\mathbf{B}_{2}
\end{array}\right]=\left[\begin{array}{c}
\mathbf{U}_{E 1}^{T} \\
\mathbf{U}_{E 2}^{T}
\end{array}\right]\left(\mathbf{W}_{o} \mathbf{B}-\mathbf{C}^{T}\right),\left[\begin{array}{ll}
\mathbf{C}_{1} & \mathbf{C}_{2}
\end{array}\right]=\left[\begin{array}{c}
\mathbf{C P} \\
-\rho \mathbf{B}^{T}
\end{array}\right]\left[\begin{array}{ll}
\mathbf{V}_{E 1} & \mathbf{V}_{E 2}
\end{array}\right],} \\
\mathbf{D}=\mathbf{D} .
\end{gathered}
$$

The Hankel approximation function is also implemented in MATLAB (MATLAB $\mathrm{Gr}=$ Hankelmr(sys,order)). Moreover, the error of the simulation is negligible; the algorithm restarts in case the error is unsatisfactory.

\section{Numerical results}

The study of the algorithms for models order reduction was carried out in MATLAB on a PC (Intel Pentium i5 CPU3825U 2x1.9GHz ROM 4Gb).

As an example, two random systems were created in the state space of the order 100 and $1000($ MATLAB sys $=$ rss(n)). By applying various algorithms for the system order reduction, Bode diagrams, which represent the frequency response of a linear stationary system on a logarithmic scale, and the step function of systems, were constructed. Engineers find the Bode diagram and the step function appealing to apply in various fields. Apart from this, the time on reduced order models generation by means of calculating the time (MATLAB tic;toc;) on the corresponding algorithm code execution, as well the time on the step function generation for each reduced order model (MATLAB tic;step(sys);toc;) are estimated.

Now, let us consider the first system of order 100. Figure 4 shows the singular values of the system. According to the figure, the system is stable and the singular numbers $\sigma_{1} \ldots \sigma_{23}$ are much larger than others, therefore, when creating a reduced order model within the SVD methods, we will use the original order 23 . We take the same order for the Krylov method.

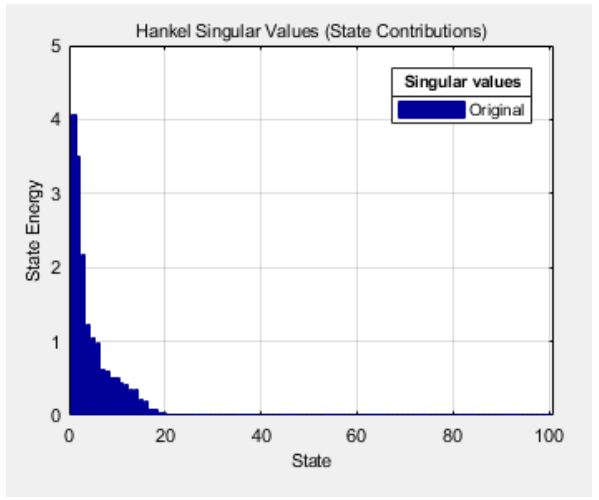

Fig. 4. Singular numbers of the original system.

Figures 5 and 6 show the Bode diagram and the step function for reduced order models 23 , respectively. 

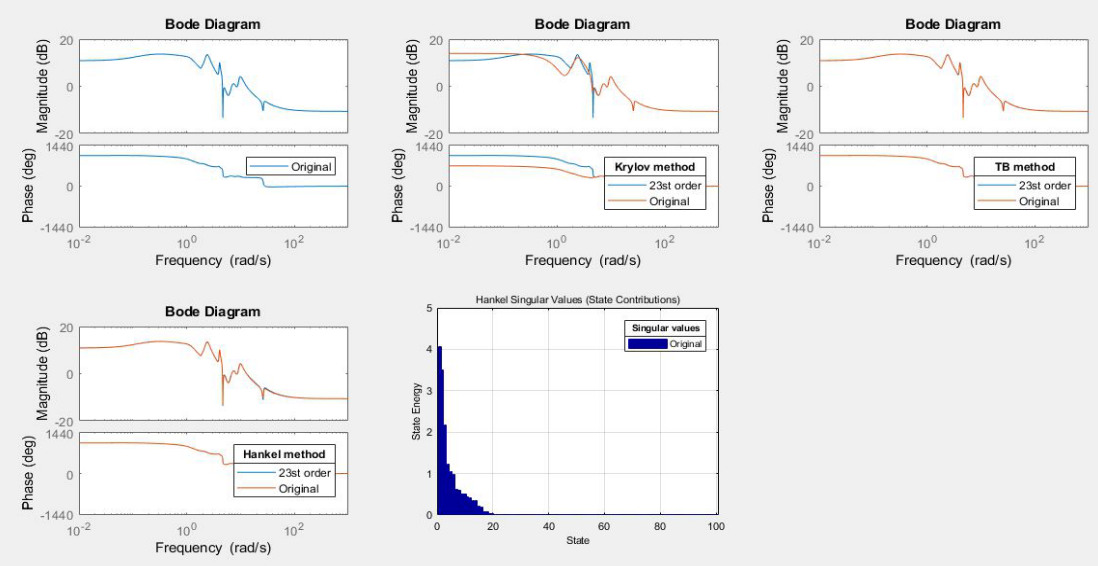

Fig. 5. The Bode diagram of order system 100 and reduced order models 23.

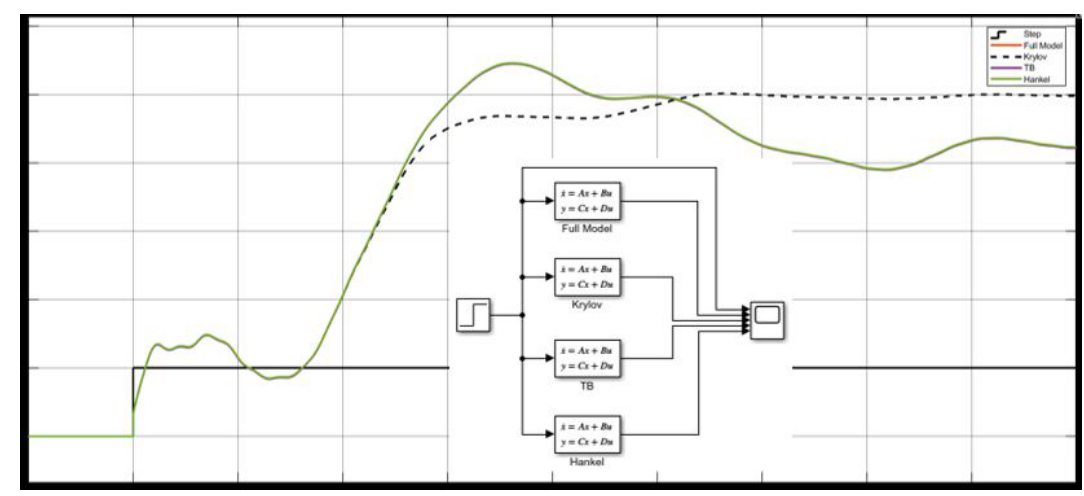

Fig. 6. Step function of order system 100 and reduced order models.

According to the graphs presented here, the Krylov algorithm (dashed line) shows unsatisfactory results as the graphs have significant discrepancies, while SVD methods provide good results. To obtain a more accurate model using the Krylov method, the system order was increased to 50 (figure 7 and 8).
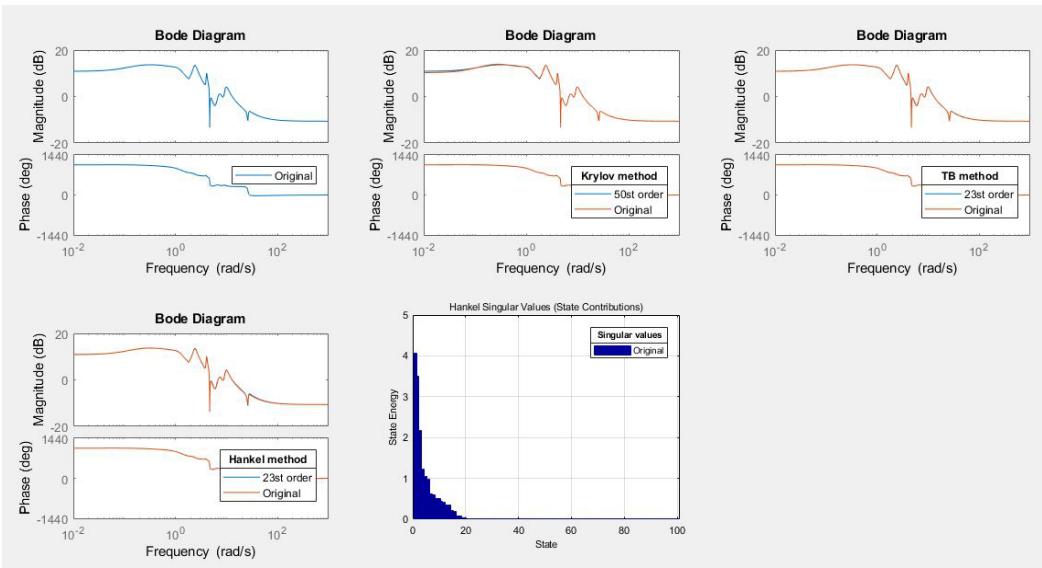

Fig. 7. The Bode diagram of order system 100 and reduced order models (Krylov method - order 50, SVD-methods - order 23). 
In the latter case, the models obtained demonstrate extremely high simulation accuracy. Table 1 shows the time spent on simulation of the reduced order model using various methods and the relative error of the step function, as well as the time on step function generation.

Thus, when using reduced order models for small order systems 100 or less, the computational efforts are almost the same.

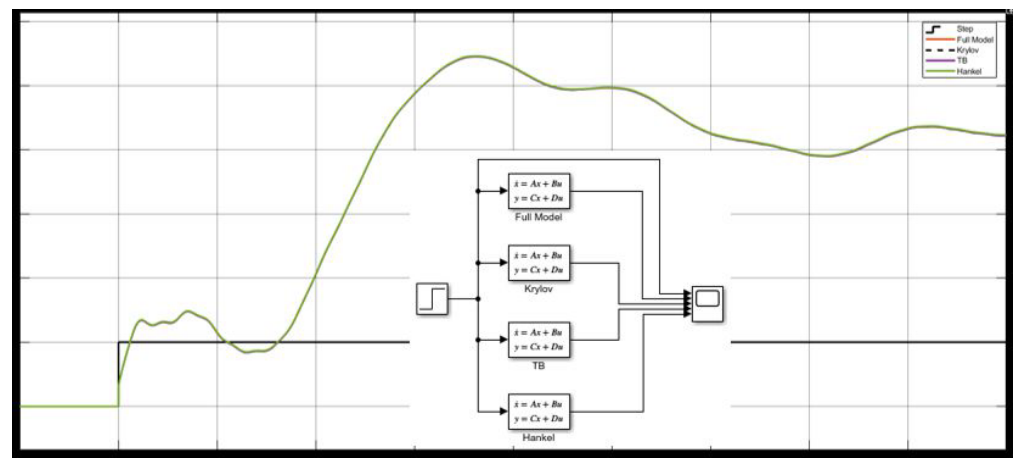

Fig. 8. Step function of order system 100 and reduced order models (Krylov method - order 50, SVDmethods - order 23).

Table 1. Time on models calculation

\begin{tabular}{|l|c|c|c|}
\hline \multicolumn{4}{|c|}{ The order of original system: 100 } \\
\hline Method & $\begin{array}{l}\text { Time on reduced order } \\
\text { model simulation, sec }\end{array}$ & $\begin{array}{l}\text { Relative } \\
\text { error, \% }\end{array}$ & $\begin{array}{l}\text { Time on step function } \\
\text { generation, sec }\end{array}$ \\
\hline Krylov subspace method 50th & 0,015 & 0,02 & 0,09 \\
\hline Balanced truncation 23th & 0,07 & 0,04 & 0,19 \\
\hline Hankel approximation 23th & 0,16 & 0,34 & 0,09 \\
\hline Original system & & & 0,35 \\
\hline
\end{tabular}

Now, let us consider a system of higher order 1000. Figure 9 shows Bode diagrams of reduced order models: the Krylov method - order 500, the balanced truncation method - order 360, the Hankel approximation method - order 545 and the singular values of the system; Figure 10 shows the step function. It should be noted that in this case the system is not stable (singular numbers of the unstable part of the system are marked in red on the graph of singular values), and in SVD methods there is a preliminary decomposition of the system into stable and unstable subsystems, followed by order reduction in the stable one. 

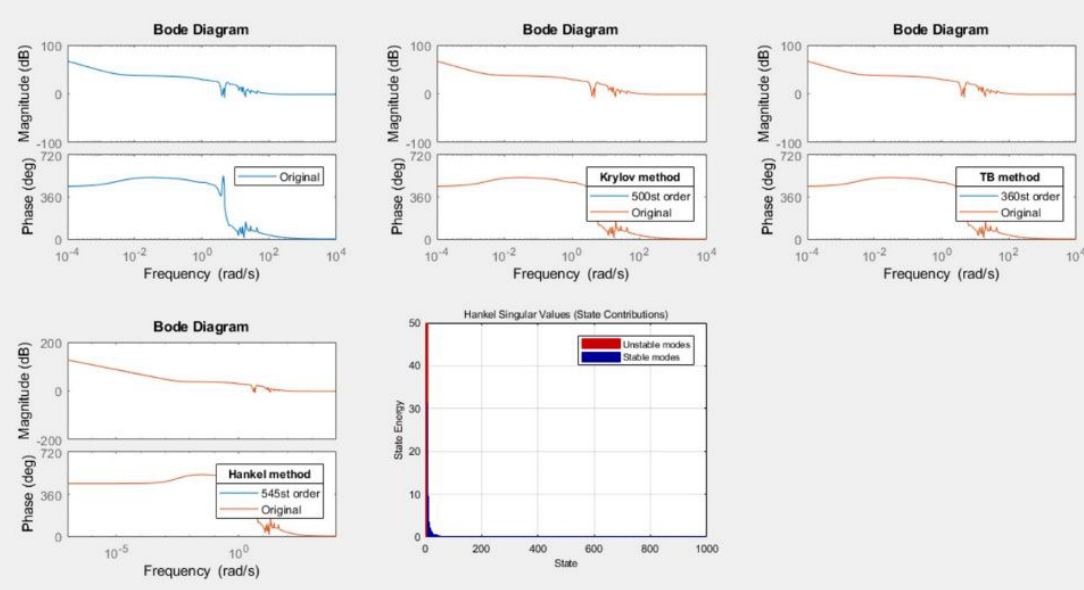

Fig. 9. The Bode diagram of order system 1000 and reduced order models.

All methods demonstrate good results. Table 2 shows the time on reduced order model simulation by various methods and the relative error of the step function, as well as the time on the step function generation.

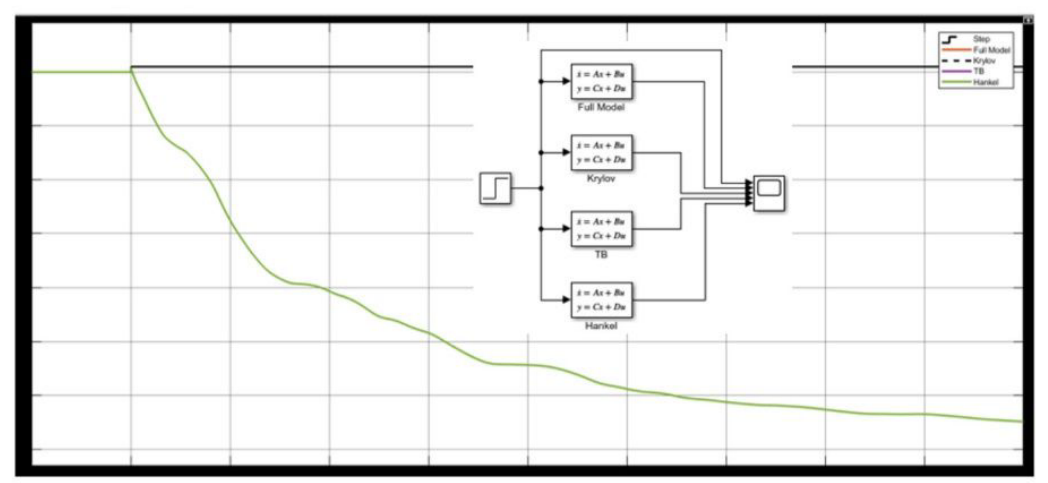

Fig. 10. Step function of order system 100 and reduced order models (Krylov method - order 500, SVD-methods - order 360, Hankel approximation - order 545).

Table 2. Time on models calculation

\begin{tabular}{|c|c|c|c|}
\hline \multicolumn{3}{|c|}{ The order of original system: 1000 } \\
\hline Method & $\begin{array}{c}\text { Time on reduced } \\
\text { order model } \\
\text { simulation, sec }\end{array}$ & $\begin{array}{c}\text { Relative error, } \\
\%\end{array}$ & $\begin{array}{c}\text { Time on step } \\
\text { function generation, } \\
\text { sec }\end{array}$ \\
\hline Krylov subspace method 500th & 1,58 & $1,7 \cdot 10^{-9}$ & 0,71 \\
\hline Balanced truncation 360th & 24 & $3,05 \cdot 10^{-12}$ & 0,73 \\
\hline Hankel approximation 545th & 159 & $3,61 \cdot 10^{-7}$ & 2,38 \\
\hline Original system & & & 9,98 \\
\hline
\end{tabular}

\section{Conclusion}

In this paper, methods for generating reduced order models of linear systems are considered. For the small dimension systems (less than 100), reduced order models do not have significant advantages in computational performances, whereas for the high dimension systems (from 1000 and more), reduced order models make it possible to reduce significantly 
the computation power (up to 10 times). The methods based on Krylov subspaces are advantageous in terms of computational efforts and can be used for high-dimension systems, however, such methods do not have algorithms for automatic estimation of global error $[9,10]$. SVD methods have the ability to estimate a global error, but they are expensive in terms of computing power. Any reduced order model, simulated by applying the order reduction methods, should be used with great care to become a good tool for optimization and adjustment of newly developed systems.

The reported study was funded by DAAD. The reported study was funded by Ministry of Education and Science of the Russian Federation. Project \#8.12811.2108/12.2. This article is based on results obtaned by using equipment of shared facility "Diagnosis and energy-efficient electrical equipment" (NPI).

\section{References}

1. A.L. Balaban, Yu.A. Bakhvalov, V.V. Grechikhin, D.V. Shaykhutdinov, J. Eng. \& App. Sci. 13(7), 1696 (2018)

2. A. Lankin, D. Shaykkhutdinov, V. Grechikhin, D. Schuchkin, N. Narakidze, N. Gorbatenko, J. Eng. \& App. Sci. 12(2), 363 (2017)

3. D. Shaykhutdinov, N. Gorbatenko, Yu. Manackov, K. Shirokov, MATEC Web of Conferences 132, 04021 (2017)

4. V.N. Lancov, Methods for reducing the order of models of complex systems (Vladimir, VlGU, 2017)

5. V.G.Kurbatov, S.D. Kurgalin, Vestnik VSU, S.: System Analysis And Information Technologies 1, 46- 54 (2013)

6. Model Reduction Routines for SLICOT. Available at: https://elib.dlr.de/3523/1/varga_nic1999-8.pdf

7. Model reduction. Available at: http://cse.lab.imtlucca.it/ bemporad/teaching/ac/pdf/AC2-07-Model_Reduction.pdf

8. M.G. Safonov, R.Y. Chiang, D.J.N. Limebeer, IEEE Trans. on Automat. Contr., 35, 496 (1990)

9. E.B Rudnyi, J. G. Korvink, Sensors Update 2, 3 (2002)

10. E.B. Rudnyi J. G. Korvink, GACMReport 2, 2 (2006) 